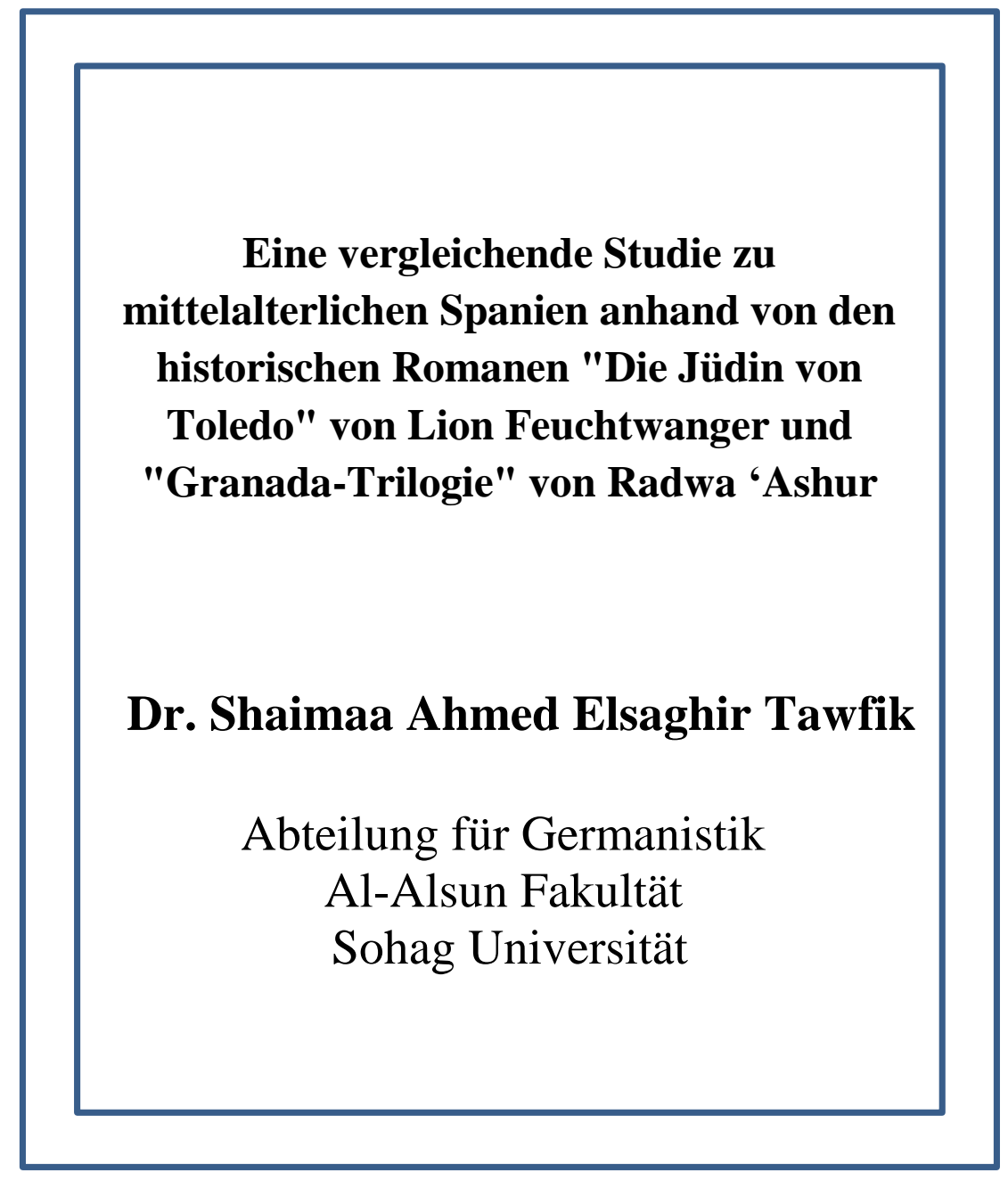



„Fern im Süd das schöne Spanien, Spanien ist mein Heimatland, Wo die schattigen Kastanien Rauschen an des Ebro Strand, Wo die Mandeln rötlich blühen, Wo die süße Traube winkt Und die Rosen schöner glühen Und das Mondlich gold'ner blinkt.“ Emanuel Geibel (1815-1884)

\section{Abstract (Deutsch)}

Wenn die Rede von der Darstellung Spaniens in der Literatur ist, dann stehen Städte wie Granada, Cordoba, Toledo, Sevilla und Málaga als Schauplätze berühmter Werke der deutschen und arabischen Literatur im Mittelpunkt. Durch die vorliegende komparatistische Untersuchung wurde der Versuch unternommen, das Augenmerk auf die moderne literarische Darstellung des mittelalterlichen Spaniens und die literarische Gattung Historischer Roman in der modernen deutschen und ägyptischen Literatur anhand der historischen Romane Die Jüdin von Toledo (1955) von Lion Feuchtwanger und GranadaTrilogie (1994) von Radwa 'Ashur zu richten. Beide 
untersuchten Romane weisen sowohl Gemeinsamkeiten als auch Unterschiede auf. Die Arbeit kann als Ansatz im Bereich der interkulturellen literarischen Diskursanalyse fungieren. In diesem Rahmen werden in den ausgewählten Texten die wichtigsten textinternen und -externen Elemente berücksichtigt. Darüber hinaus werden ihre interkulturellen Vorgaben beleuchtet und analysiert.

Schlüsselwörter: Spanien, Feuchtwanger, Die Jüdin von Toledo, 'Ashur, Granada Trilogie.

\section{Abstract (Englisch)}

When talking about the representation of Spain in literature, cities such as Granada, Cordoba, Toledo, Seville and Málaga take centre stage as settings of famous works of German and Arabic literature. Through this comparative study, an attempt was made to focus attention on the modern literary representation of medieval Spain and the literary genre of the historical novel in modern German and Egyptian literature through the historical novels The Jewess of Toledo (1955) by Lion Feuchtwanger and Granada Trilogy (1994) by Radwa 'Ashur. Both novels under investigation have similarities as well as differences. The work can function as an approach in the field of intercultural literary discourse analysis. Within this framework, the most important elements internal and external to 
the text are considered in the selected texts. Furthermore, their intercultural specifications are illuminated and analysed.

\section{الملخص باللغة العربية}

هدفت الدراسة إلى التعرف على إسبانيا في العصور الوسطي من خلال الرواية التاريخية المعاصرة، من خلال هذا التحقيق المقارن، بُذلت محاولة للفت الانتباه إلى هي العرض الأدبي الحديث لموضوع إسبانيا في العصور الوسطى وكذلك مكانة الرواية التاريخية في الأدبين الألماني والعربي المعاصر وذلك من خلال دراسة مقارنة لرواية يهودية طليطلة (1955) للكاتب الألماني الكبير ليون فويشت فانغر وثلاثية غرناطة (1994) للكاتبة الأشهر في مصر والعالم العربي رضوى عاشور. وقد تم تقسيم الدراسة إلى ثلاثة فصول، تناول الفصل الأول مقدمة الدراسة، وهدفها، ومنهجيتها، والفصل الثاني عبارة عن مبحثين، عرضت الدراسة في المبحث الأول مفهوم الرواية التاريخية في اللغة الألمانية واللغة العربية وعرجت علي أهم السمات المميزة لهذا الجنس الأدبي وأوجه الثبه والتمايز بين الرواية التاريخية في الأدب الألماني و الأدب

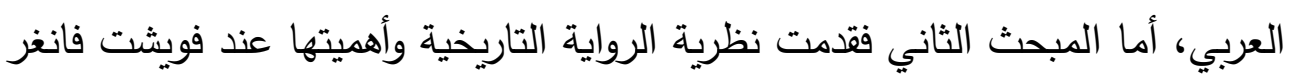
و رضوي عاشور، أما الفصل الثالث والأخير فقد كان عبارة عن مبحثين، وضحت الرواله الدراسة في المبحث الأول تحليلا سياسيا اجتماعيا لرواية يهودية طليطلة وثلاثية غرناطة، ثم في المبحث الثاني تتاولت الدراسة كيف عرض كل من الكاتب ليون فويشت فانغر والكاتبة رضوي عاشور صورة إسبانيا في العصور الوسطي وعلاقتها بالتاريخ المعاصر، وأخيرا قدمت الدراسة أهم النتائج، وطرحت قائمة بالمراجع والمصادر التي استعانت بها؛ لتقديم العمل حتي نهايته. 


\section{Einleitung}

Für die vorliegende Studie ist vor allem von Bedeutung, wie Lion Feuchtwanger und Radwa 'Ashur durch eine historische Konstruktion ein zeitloses Spanienbild schaffen, das nicht nur mit politischen Grenzen verbunden ist, sondern auch vertretungsweise Widerstandsmechanismen in der modernen Gesellschaft aufweist.

Die am 26. Mai 1946 in Kairo geborene Radwa 'Aschur gilt als eine der meistgelesenen Autorinnen in Ägypten. Sie war als Professorin für komparatistische Literatur an der Abteilung für Anglistik der Ain-Schams-Universität tätig. Zudem war sie Gastprofessorin an verschiedenen arabischen, amerikanischen und europäischen Universitäten (vgl. Mohamed, 2015, S. 276). Radwa 'Aschur begann ihre Karriere als Schriftstellerin in den 80er-Jahren und wurde in kurzer Zeit eine der angesehenen Schriftstellerin nicht nur in Ägypten, sondern im gesamten arabischen Sprachraum. Dieser Erfolg brachte Übersetzer und Verleger dazu, ihre $\mathrm{g}$ Romane, Kurzgeschichten und Literaturkritiken in verschiedenen Sprachen wie z. B. Englisch, Spanisch, Italienisch und Indonesisch, zu übersetzen und zu veröffentlichen.

In ihrem Roman Granada-Trilogie stellt die Autorin die Geschichte mehrerer Generationen einer muslimischen 
arabischen Familie dar, die in Granada lebt, angefangen von der Belagerung und Niederlage des nasridischen Königreichs Granada im Winter 1491 bis zur endgültigen Vertreibung der Moriscos (der Muslime im Spanien des 16. Jahrhunderts, die 1609 unfreiwillig zum Christentum konvertiert waren). Die Autorin hat mit dem Titel Granada als ,,verlorenes Paradies“ (al-firdaws al-mafqūd) symbolisiert (vgl. Ojeda Arnal, 2019, S. 306).

Die Trilogie (Thulalaythiyat Gharnata), die einen Umfang von 502 Seiten hat, besteht aus drei Romanen. Granada, Maryama und Der Auszug lauten die Titel der Romane der Trilogie, die 1994 erstmals in Arabisch erschienen ist. Des Weiteren sind u. a. Übersetzungen ins Englische und Spanische für nichtarabische Leser vorhanden, was den Stellenwert der ägyptischen Autorin in der internationalen Literatur nachweist.

Das Werk Die Jüdin von Toledo oder Spanische Ballade (1955) von dem deutschen Schriftsteller Lion Feuchtwanger, das zu den bemerkenswertesten der deutschsprachigen Gegenwartsliteratur zählte, ist ein historischer Roman, mit dem Feuchtwanger das kulturelle, politische und religiöse Spanienbild als Gegenstand eingeführt hat. Mit demselben Thema wie bei Radwa 'Aschur setzt er sich in den 50er-Jahren auseinander. Feuchtwanger skizziert in diesem Roman die gesellschaftlichen, 813 
politischen und interkulturellen Ereignisse im Toledo des 12. Jahrhunderts. Im Mittelpunkt der Romanhandlung steht der König Alfonso VIII. und seine Beziehung zu Raquel, der Tochter seines jüdischen Schatzkanzlers Jahuda Ibn Isra.

Die Gattung Historischer Roman im Deutschen und im Arabischen

Es gibt kaum eine literarische Gattung, die sich den medialen Verwertungsinteressen so sehr unterworfen hat wie der Geschichtsroman (vgl. Aust, 1994, S. 1). Dementsprechend erweist diese literarische Gattung sich als optimaler Träger und attraktives Kostüm von Bildung, Ideologie und Wissen.

Um einen Überblick zu Feuchtwangers Roman Die Jüdin von Toledo und 'Aschurs Granada-Trilogie zu schaffen, sollte zunächst der Begriff Historischer Roman im Deutschen sowie im Arabischen erklärt werden. Zudem sollte auch die Stellung dieses literarischen Genres im deutschen und arabischen Sprachraum eruiert werden.

Mit dem Begriff „Historischer Roman“ wird ein Romantypus bezeichnet, in dem eine teilweise fiktive Handlung mit realer Geschichte verbunden ist. Dementsprechend basiert diese literarische Gattung auf der deutlichen Integration von realen und fiktiven Handlungsbestandteilen sowie literarischen und historiographischen Darstellungstechniken (vgl.Nünning, 
2013 , S. 318). Zudem kann dieser Begriff auf unterschiedliche Weise definiert werden. Der historische Roman wird im Allgemeinen verstanden als eine ,,umfangreichere, erzählende Dichtung, die beglaubigte geschichtliche Persönlichkeiten oder Tatsachen zum Gegenstand hat" (Nussberger \& Kohlschmidt in Ofner, 2004, S. 33). Eine traditionelle Definition der Gattung historischer Roman, die ähnlich wie der Erklärungsansatz von Nussberger und Kohlschmidt ist, stellt diesen als einen Roman dar, in dem historische Personen und Ereignisse auftauchen. Ihr zufolge kann der historische Roman auf erfundenen Charakteren basieren, um eine vergangene Epoche zu eruieren (vgl. Gallmeister, 1991, S. 160).

Eine konkrete Auslegung für das literarische Genre Historischer Roman im Arabischen ist schwierig zu finden. Die Problematik liegt darin, dass es eine Herausforderung ist, genaue Definitionen in Bereichen der Geisteswissenschaften zu finden. Eine der wichtigsten Definitionen, die in diesem Zusammenhang erwähnt werden kann, stammt aus dem Wörterbuch zeitgenössischer literarischer Begriffe:

Storytelling basiert auf historischen Fakten, die in modernen Schriften mit einer kognitiven Dimension eingeführt werden, und neigt dazu, eine didaktische und pädagogische Funktion 
zu etablieren. (Wörterbuch zeitgenössischer literarischer Begriffe, S. 103)

Eine weitere Begriffsbestimmung, die das Wörterbuch arabischer Begriffe in Sprache und Literatur vorstellt, ist, dass der historische Roman ein auktoriales Erzählen über historische Ereignisse enthält, die bereits eingetreten sind, mit dem Versuch, eine historische Periode mit realen oder imaginären Charakteren oder beiden wiederzubeleben (vgl. Wörterbuch der arabischen Terminologie in Sprache und Literatur, in Tabeel, 2016, S. 3).

'Abd al-Hamīd Qițt ist der Meinung, dass es einen neuen Historischen Roman gibt, den er als Kunstwerk bezeichnet, in dem die Geschichte als Stoff verwendet wird, um die Vision und Stellungnahmen der Autor darzustellen (vgl. Qitt, 1982, S. 31).

Anhand dieser Erklärungen des Begriffs Historischer Roman kann man erschließen, dass die Definition dieser literarischen Gattung im Arabischen trotz kleinerer Unterschiede weitgehend mit der im Deutschen übereinstimmt, da es in beiden Sprachen um eine authentische Geschichte und teilweise reale Personen geht. Das Interesse an der Darstellung der Geschichte, reale Figuren und vor allem authentische Schauplätze erweist sich in diesem Zusammenhang als wesentliches Prinzip in den beiden Sprachen. Die deutschen 
und arabischen Historischen Romane sind auch durch das auktoriale Erzählen gekennzeichnet, dadurch erschient im Mittelpunkt der Handlung explizit ein Erzähler, der sich an den Leser*innen direkt orientiert. Eine weitere Ähnlichkeit ist, dass der historische Roman in der deutschen und arabischen Gegenwartsliteratur Geschichtssignale aussendet. Demzufolge beziehen sich die Autor*innen auf amtliche Dokumente, Namen von Personen, Epochen, Ereignissen und Städten sowie kulturelle und geschichtliche Einzelheiten.

Zuletzt weist der historische Roman im Deutschen und Arabischen Gemeinsamkeiten in der Versetzung der Vergangenheit in die Fiktion und der Verwendung der temporalen Individualisierung der Sprache auf.

Die Theorie des historischen Romans bei Lion Feuchtwanger und Radwa 'Aschur

Der Schriftsteller Lion Feuchtwanger zählt von 1923 bis in die Gegenwart zu den wichtigsten Autoren Historischer Romane. (vgl. Aust 2016, S. 121). Er setzte sich intensiv mit der Theorie des historischen Romans auseinander und widmete sich der Literaturkritik in vielen Beiträgen, Vorträgen und Artikeln, die den Stellenwert dieses literarischen Genres eruiert haben, darunter sein Pariser Vortrag ,,vom Sinn und Unsinn des historischen Romans“ (1935) und die Monografie Das Haus der Desdemona 817 
(1961) (vgl. ebenda). Feuchtwanger als exilierter Autor war davon überzeugt, dass die historische Dichtung eine der wichtigsten und interessanten Formen des literarischen Kanons sei. Deshalb rückt das literarische Genre des Historischen Romanes ins Zentrum seines literarischen Schaffens, darunter die Josephus-Trilogie, Jud Süß, Die hässliche Herzogin Margarete Maultasch, Der falsche Nero, Waffen für Amerika, Die Füchse im Weinberg, Goya oder der arge Weg der Erkenntnis, Narrenweisheit oder Tod und Verklärung des Jean-Jacques Rousseau, Die Jüdin von Toledo und Jefta und seine Tochter.

Der Historische Roman der Weimarer Republik weist zwei wesentliche Merkmale auf: ,die völkische Blut-und-BodenIdeologie und eine religiöse Innerlichkeit" (Westenfelder 1989, in Aust, 2016, S. 113). Laut Hugo Aust ist Feuchtwanger ,ein historischer Romancier mehrerer Epochen" in der Zeit der Weimarer Republik, trotzdem war er der einzige Autor unter seinen Zeitgenössischen, der die herrschende antidemokratische Tendenz in seinen historischen Roman vermeiden konnte (vgl. Aust 2016, S. 113).

Lion Feuchtwangers Theorie des Historischen Romans basiert auf seiner Vorstellung, dass Historische Romane eine angemessene Waffe gegen Gewalt und Dummheit sind (vgl. Feuchtwanger, in Dietschreit, 1988, S. 89). Dabei versteht er 
dieses Genre als einen Roman, der die Vergangenheit neu revitalisiert, zudem verschmilzt er die Vergangenheit mit der aktuellen Gegenwart in einem literarischen Werk (vgl. Aust, 2016, S. 122). Feuchtwangers Theorie bezieht sich auf drei Grundbegriffe der Romanpoetik, nämlich Distanz, Erlebnis und Gestaltung. Diese drei Konzepte sind auch bei berühmten Autoren, Kritikern und Philosophen wie z. B. Lukács, Scott, Brecht und Nietzsche vorhanden.

Radwa 'Aschur hat aktiv an der Entwicklung der inhaltlichen und strukturellen Form des Historischen Romans in der modernen arabischen Literatur mitgewirkt. 'Aschurs Faszination und Auseinandersetzung mit der literarischen Gattung Historischer Roman ist ein wesentliches Element in den meisten ihrer Werke, ob kreativ oder kritisch. Sie äußert dazu: ", the fact that there is no novel outside history is indisputable“ ('Aschur, in Hanafy, 2020, S.3). Laut Hucheon zeigt 'Aschurs Narrativität verschiedene Aspekte, die üblicherweise der postmodernen Fiktion oder der historiographischen Metafiktion zugeschrieben werden (vgl. Hucheon, in Hanafy, 2020, S. 23). 'Aschurs Theorie des Historischen Romans bezieht sich auf die theoretischen Grundlagen von Georg Lukács, der selbst von dem in Paris gehaltenen Vortrag ,,von Sinn und Unsinn des historischen Romans“ von Lion Feuchtwanger inspiriert wurde: 
Without a felt relationship to the present, a portrayal of history is impossible. But this relationship, in the case of really great historical art, does not consist in alluding to contemporary events, [....] but in bringing the past to life as the prehistory of the present, in giving poetic life to those historical, social and human forces which in the course of a long evolution, have made our present-day life what it is and as we experience it (Lukacs 1983, S. 53).

Dabei hat sie eine weitere Dimension der doppelten narrativen Perspektive, die in der Metafiktion als Instrument benutzt wird, um den Begriff des tyrannischen Verfassens oder die Verlebung der Vergangenheit zu problematisieren. Laut der vom Döblin dargestellten Theorie ,Mit Historie will man was“ (Döblin 1936, S. 172 in Aust, 2016, S. 122) stellt Radwa 'Aschur sich eine Geschichte der Niederlage vor, und im Reich der Imagination schreibt sie Raum, Zeit, kollektives Gedächtnis und kulturelles Erbe neu ein. 'Aschur wiederholt die Artikulationen der Selbstheit einer Gemeinschaft, indem sie die Fiktion als Werkzeug benutzt, um die reichen inneren Leben, die Gedanken, die Emotionen, die privaten und intimen Räume zu rekonstruieren, die die Geschichtsschreibung nie vollständig berücksichtigen konnte. 
Zusammengefasst kann erschlossen werden, dass Lion Feuchtwanger und Radwa 'Aschur in starkem Maß das literarische Genre des Historischen Romans beeinflusst haben. Die deutsche sowie die arabische Gegenwartsliteratur sind innen dankbar, dass die beiden Autor*innen dieses Genre wieder auf die Bestsellerliste der Belletristik im deutschen und arabischen Sprachraum geführt haben.

Feuchtwangers und 'Aschurs politisch-sozialkritische Dimensionen in den beiden Romanen

Feuchtwangers und 'Aschurs Auseinandersetzungen drehen sich um zwei Grundprobleme: die Fiktionalität historischer Ereignisse und die Übermittlung zeitgenössischer Themen im historischen Kontext. Auf der einen Seite hat 'Aschur in ihrer Trilogie eine erfundene andalusischen muslimischen Familie im Rahmen des historischen Kontextes dargestellt, dementsprechend ist der Roman ,,eine fantastische Geschichte innerhalb der objektiven Geschichte“ (vgl. Al-Alam 1993, S. 314). Obwohl diese Trilogie zu der literarischen Gattung Historischer Roman gehört, hat Radwa 'Aschur keine historischen Figuren als Hauptfiguren im Fokus der Romanhandlung vorgestellt. Dagegen stehen normale Figuren mit ihrem Alltag im Vordergrund. Der Grund dafür ist, dass der 
Text metaphorische Bedeutungen enthält. Dies bestätigt Radwa selbst im Vorwort ihrer Trilogie:

Der Roman behandelt nicht das Leben von Königen und Politikern, sondern den Alltag von normalen Menschen, die die Zeit der Reconquista erleben mussten. Der Text verbindet die Geschichte dieser Zeit mit den Details des Lebens einer arabischen Familie aus Granada. ('Aschur, 1994, S. 314)

Auf der anderen Seite beschäftigt sich Lion Feuchtwanger in seinem Roman mit der Darstellung des Königs Alfonso VIII. von Kastilien und der Jüdin Raquel. Im Gegensatz zu Radwa 'Aschur wählt er seine Figuren aus dem höfischen Milieu, zudem stehen die historischen, politischen und gesellschaftlichen Ereignisse im damaligen Spanien direkt im Fokus der Romanhandlung. Bei Lion Feuchtwanger und Radwa 'Aschur kommt das mittelalterliche Spanien vor, als eine Stadt, die durch die drei Religionen Islam, Christentum und Judentum geprägt ist. Feuchtwanger und Radwa bestätigen damit, dass in der Zeit der islamischen Eroberung die drei Religionen in Frieden lebten. Über das glückliche Leben, das die Juden damals in Spanien erlebten, hat Feuchtwanger folgendes geäußert: 
Den Unterworfenen zeigten die Moslems Milde. Für ihre Christen übertrugen sie das Evangelium ins Arabische. Den zahlreichen Juden, die von den christlichen Westgoten unter strenges Ausnahmerecht gestellt worden waren, räumten sie bürgerliche Gleichheit ein. Ja, es führten unter der Herrschaft des Islams die Juden in Spanien ein so glückhaft erfülltes Leben wie niemals vorher seit dem Unt ergange ihres eignen Reiches. (Feuchtwanger 2018, S. 10)

Radwa 'Aschurs Granada-Trilogie und Feuchtwangers Die Jüdin von Toledo nutzen die Kunst der Erzählung, um die gegenständliche und wörtliche Leere, die dem verkleinerten, dämonisierten und entmenschlichten kolonialen Subjekt zugeschrieben wird, zu problematisieren und herauszufordern. Feuchtwanger erzählt seine fiktiven Welten aus den Augen, Ohren und Herzen jüdischer Individuen und im aktuellen politischen Kontext, während auf der anderen Seite 'Aschur aus den Augen, Ohren und Herzen muslimischer Individuen den Plot ihres Romanes konzipiert. Diese Geste schafft einen dringend notwendigen Dialog zwischen einer imaginierten Vergangenheit, der Erfahrung des Lesers der Gegenwart und natürlich der potenziellen Zukunft.

Im folgenden Abschnitt geht es um, wie Lion Feuchtwanger durch seinen Roman Die Jüdin von Toledo und 823 
Radwa 'Aschur durch inre Granada-Trilogie das Bild des mediävalen Spanien in Postkolonialismus und in der postmodernen Literatur dargestellt haben.

\section{Das mittelalterliche Spanien bei Lion Feuchtwanger}

\section{und Radwa 'Aschur}

Das mediävale Spanien hat eine besondere Anziehungskraft, die nicht nur deutsche und arabische Schriftsteller*innen, sondern auch Weltautor*innen angelockt hat, um es und seine Geschichte als Basisstoff für verschiedene wichtige Werke der Belletristik auszusuchen. Das mittelalterliche Spanien ist durch seine damalige multiethnische Gesellschaftsstruktur gekennzeichnet. Damit konnte es eine höchste kulturelle Blüte erreichen. Aber hinter den Kulissen gab es immer Konflikte, Kriege, Rivalität und Alterität, was sich am deutlichsten in den vorliegenden untersuchten Werken manifestiert.

Feuchtwanger hat Spanien zum zweiten Mal für seinen Roman Die Jüdin von Toledo ausgesucht, da es schon zuvor im Jahr 1948 zum ersten Mal in seinem Roman Goya oder der arge Weg der Erkenntnis als Hintergrund auftaucht (vgl. Gelhard 2008, S. 91). In seinem zweiteiligen Roman Die Jüdin von Toledo oder spanische Ballade hat Feuchtwanger ein ausgedehntes Bild der Begegnung der drei Offenbarungsreligionen - Islam, Christentum und Judentum - 
und Kulturen im mediävalen Spanien im 12. Jahrhundert in der Regierungszeit von König Alfons VIII. vorgestellt. Er bezweckt mit diesem Roman, das Leben in Spanien zu der jeweiligen Zeit zu eruieren. In einem Brief an Arnold Zweig vom 20. Juli 1953 hat er sich so geäußert:

Der innere Sinn ist die Darstellung der ungeheurn Anziehungskraft des Krieges, der sich nicht einmal die Gegner ganz verschließen können. Darstellen will ich also, welch ungeheure Widerstände der Kampf um den Frieden überkommen muß. Das Schicksal meines jüdischen Ministers Jehuda Ibn Esra wiederholt auf einer sehr viel höheren geistigen Ebene das Schicksals Jud Süß (Feuchtwanger 2018, Vorwort).

In diesem Roman setzt sich Feuchtwanger mit der Vergangenheit der iberischen Halbinsel auseinander. Darüber hinaus hat er sich nicht ausschließlich mit historischen Gestalten befasst, sondern auch mit anderen Themen, wie z. B. dem konfliktgeprägten Zusammenleben von Islam, Judentum und Christentum, Fremde, Identität, Liebe und Politik.

Die Liebesgeschichte zwischen König Alfons VIII. und der Jüdin Raquel ist eine reale Geschichte, die viele Autoren wie z. B. Franz Grillparzer, Lion Feuchtwanger, Abraham S. Marrache und Lope de Vegas inspiriert hat. Diese Geschichte geht auf 
das biblische Buch zurück, in dem die Geschichte der schönen Esther erzählt wurde. Das Buch Esther hat Feuchtwanger sehr angerührt, deshalb legt er diese Geschichte seinem Text zugrunde. In seinem Nachwort zu dem Roman äußert er:

,Mich jedenfalls hat »Das Buch Esther« tief angerührt, es hat viele angerührt, und in den mehr als zweitausend Jahren seit seiner Entstehung haben viele versucht, den Roman aus den Geschehnissen ihrer eignen Zeit heraus zu erzählen. Mehrmals, wenn ich die Bedrängnis der beiden Völker, deren Verband ich angehöre, besonders schmerzhaft spürte, hat es auch mich getrieben, aus dem Sehwinkel meiner Welt heraus die Geschichte der Königin Esther neu zu erzählen (Feuchtwanger 2018, S. 501).

Feuchtwanger hat in Spanien einen Schauplatz für seinen historischen Roman gefunden. Er war sowohl von dem Rittertum zur Zeit der Kreuzzüge als auch ,,von den Kämpfen zwischen Christen und Moslems um die Vorherrschaft auf der Iberischen Halbinsel“ (Gelhard 2008, S. 92) und deren unterschiedlichen Lebenskonzepte fasziniert.

Spanien erlebte unter die Herrschaft der Mauren seine Blütezeit und wurde zum wichtigsten Kulturzentrum Europas:

,Künste und Wissenschaften blühten wie bisher niemals unter diesem Himmel. Erhabenes und Zierliches mischten 
sich, die Häuser auf besondere, bedeutende Art zu schmücken. Ein kunstvoll verästeltes Erziehungssystem erlaubte einem jeden, sich zu bilden. Die Stadt Córdova hatte dreitausend Schulen, jede größere Stadt hatte ihre Universität, es gab Bibliotheken wie niemals seit der Blüte des hellenischen Alexandria (Feuchtwanger, S. 10).

Man geht davon aus, dass Feuchtwanger eine positive Stellungnahme zu dem Islam und Judentum abgibt, auf der anderen Seite stellt er das Christentum als kriegerisch, kalkulierend und kalt dar.

Im Roman Die Jüdin von Toledo geht es um ein wichtiges Anliegen Feuchtwangers: den Leser in das frühmittelalterliche Spanien des 12. Jahrhunderts zu führen. Im Mittelpunkt der Handlung taucht der junge christliche König von Kastilien Alfons VIII. auf, der wegen seines leichtsinnigen Feldzuges gegen die Moslems sein Land in riesige Not gebracht hat. Um seine finanzielle Lage zu verbessern und Kastilien wieder aufblühen zu lassen, tritt der Kaufmann aus Sevilla Ibrahim (ein Mann jüdischer Herkunft namens Jehuda) auf, um das Amt des Finanzministers in Toledo zu übernehmen. Anhand des Titels des Romans soll der Ort der Handlung Toledo sein. In der Residenz des Königreichs Kastilien, Burgos, Galiana und im Hause Jehudas spielen fast alle Szenen des Romans. 
Seit dem 11. Jahrhundert, als die erste andalusische Stadt (Toledo) unter kastilische Herrschaft fiel, hat Spanien ebenfalls eine bedeutende Bedeutung in der arabischen Literaturtradition, sei es durch Trauergedichte von andalusischen Dichtern der Zeit oder moderne Dichter der arabischen Gegenwartsliteratur wie Ahmed Shawki ,Nizar Qabbani und Mahmoud Darwish, die sich mit Al-Andalus'-Nostalgie beschäftigen (vgl. Louayene 2013, S. 36).

In ihrer Trilogie rekonstruiert Radwa 'Aschur die Zeit und den Moment nach 1492, um zu zeigen, wie Muslime und ihre Nachkommen nach der Eroberung Granadas nicht einfach von der iberischen Halbinsel verschwunden sind. Dieser historische Moment von 1492 kann oft als Endpunkt für den Islam in Spanien und als Ausgangspunkt für die europäische Expansion in die "neue Welt" gedacht werden. Dadurch hat Radwa Spanien als Land vorgestellt, das an der Schwelle einer großen sozialen Umwälzung ist (vgl. Hanafy 2020, S. 3). 'Aschur erzählt, dass ihr Interesse an dem Thema plötzlich geweckt wurde, als sie im Januar 1991 während des ersten Irak-Krieges Fernsehbilder sah. Diese Bilder haben etwas mit der nackten Frau am Anfang des Romans zu tun (Übergang des Todes und des Verlusts von Granada). Dieses Bild öffnete die Tür der Erinnerung an die israelischen Bombenangriffe auf den Sinai 
1956, an die Katastrophe von 1967, an die Bombenangriffe auf den Libanon 1978 und 1982 und an die Bombenangriffe auf palästinensische Lager. Nachdem sie sich von dem Bild überredet fühlt, beginnt die Autorin, über Granada zu lesen, aber sie interessiert sich für den Alltag der Einwohner der Stadt und für die intimen Details dieser Geschichte (vgl. Garad 2014, S.94).

Mit diesem Roman führt Radwa 'Aschur die Geschichte einer arabisch-andalusischen Familie ein, die sich in einem spezifischen historischen Kontext ihrem Schicksal stellt: dem Fall Granadas, der letzten Burg der muslimischen Araber, dem Verlust Andalusiens, das seitdem ein „,verlorenes Paradies“ darstellt, und dem Verschwinden einer Zivilisation nach fast acht Jahrhunderten des Wiederaufbaus, die immer noch in dem Gedächtnis ihrer Gelehrten und Historiker ist, die ihre Phasen und Hintergründe kennen. Darüber hinaus repräsentiert Radwa sowohl eine Erfahrung des unterschiedlichen Zusammenlebens als auch eine Erfahrung, die in der Vergangenheit nicht bekannt ist. Die Handlung von Granada-Trilogie basiert auf binärer Unterdrückung und Widerstand, mit einer Vision für die Zukunft, die den Widerstand in der Gegenwart antreibt. Denn Geschichte ist nicht nur Vergangenheit, sondern auch aktive und interaktive Bausteine, die die alltägliche Gegenwart formen. 
Das Bild von dem mediävalen Spanien zwischen 1492 und 1527, das Radwa 'Aschur dem heutigen Leser vorgestellt, bezieht sich auf eine alte Landkarte von Spanien (vgl. Garad, 2014, S. 94).

Es ist bemerkenswert, dass der Ort der Handlung beschränkt wurde: fast alle Szenen des Romans spielen in Granada. Im ersten Kapitel des ersten Romans der Trilogie Granada nennt die Autorin die Orte ihrer Geschichte: Río Genil, Río Darro, Alhambra, Albaicín (das ehemalige maurische Wohnviertel) und das Haus Ibn Jafers. Diese Selbstbeschränkung der Schriftstellerin hat aber kaum die Ausdruckskraft der dargestellten Epoche zwischen 1492 und 1527 verringert: Es ist eine lebendige, plastische, unerhört suggestiv und klare, sozusagen greifbare Vision. Alle Merkmale der Epik und des dramatischen historischen Romans leuchten in dieser Trilogie in hellstem Licht. Der Ort in der Trilogie steht im Mittelpunkt des Erzählens bei 'Aschur. Die Autorin beschäftigt sich in dem ersten Roman Granada hauptsächlich mit der Stadt, wo die Protagonisten sich mit dem Umsturz Granadas, dem Verlust sowohl ihrer arabischen Identität als auch ihrer Religion auseinandersetzen.

Granada zeigt eine muslimische Stadt, eingebettet in Erinnerungen an Generationen von Leben und Tod, und die 
Besatzungstruppen von König Ferdinand und Königin Isabel werden als ausgesprochen fremd dargestellt. Die räumlichen Auslöschungen von Mauren und Morisken in der historischen Besetzung, Konversion, Zerstreuung und schließlich Vertreibung aus dem expandierenden spanischen Imperium sind ein langwieriger Prozess, das Andere verschwinden zu lassen, während nur einige der Räume und kunstvollen Umgebungen, die sie einst bewohnten, übrig bleiben.

\section{Fazit}

In der vorliegenden Untersuchung wurde der Versuch unternommen, das Augenmerk auf die Darstellung des mittelalterlichen Spanien bei dem deutschen jüdischen Schriftsteller Lion Feuchtwanger im Vergleich zu der weltweit bekannten ägyptischen Autorin Radwa 'Aschur am Beispiel von zwei von ihren wichtigsten historischen Werken, Die Jüdin von Toledo und Granada-Trilogie zu richten. Dabei wurde das Ziel verfolgt, einen Blick auf das literarische Genre Historischer Roman in Deutschland und im arabischen Sprachraum zu werfen. Aufgrund dieser Ausführung kann der/die Leser*in davon ausgehen, dass das Interesse an der Darstellung der Geschichte, reale Figuren und vor allem authentische Schauplätze sich in diesem Zusammenhang als wesentliches Prinzip in den beiden Sprachen erweisen. Laut meinem Beitrag 
geht der/die Leser*in davon aus, dass die literarische Gattung des Historischen Romans im arabischen Raum schon geschätzt wurde.

Um die Hypothese der Studie ,,Die Stellung des mittelalterlichen Spanien in den Werken von Lion Feuchtwanger und Radwa 'Aschur" zu prüfen, befasste sich der empirische Teil der Arbeit mit den Werken Die Jüdin von Toledo und Granada-Trilogie. Hierbei gehe ich davon aus, dass Lion Feuchtwanger und Radwa 'Aschur in den beiden Werken das Thema der spanischen Legende behandeln, ein Thema, das von vielen Autoren verschiedener Nationalitäten untersucht wird, insbesondere Inquisition, Zwangsbekehrung, Vertreibung usw. Die beiden Autoren interessieren sich jedoch für die Geschichte von Al Ándalus, aber in den jeweiligen Werken verwenden sie das historische Thema, um das Imagotypus nicht nur des Spanischen, sondern aller anderen zu repräsentieren. Darüber hinaus schildern die beiden Autoren mithilfe der vorliegenden Werke, inwieweit das damalige mediävale Spanien in der Zeit der kastilischen Herrschaft unter Autorität, Grausamkeit, Intoleranz, Fanatismus, Stolz, Verachtung und Hass litt. Infolgedessen führen, je nach der Sichtweise der Autoren, alle diese Fakten zu Dummheit und Wahnsinn. 


\section{Literaturverzeichnis}

\section{Deutsche und englische Literatur}

Aust, H. (1994). Der historische Roman und seine Formen. In: Der historische Roman (pp. 22-34). JB Metzler, Stuttgart.

Aust, H. (2016). Der historische Roman. Springer-Verlag

Börner, F. (2016). Auf der Suche nach Spanien (Doctoral dissertation, Universitat de Barcelona).

Dietschreit, F. (1988). Lion Feuchtwanger. JB Metzler.

Feuchtwanger, L. (2018). Die Jüdin von Toledo: Roman. Aufbau Taschenbuch.

Gallmeister, P. (1991). Der historische Roman. Formen der Literatur in Einzeldarstellungen. Hrsg. v. Otto Knörrich, 2, 160-170.

Garad, K. (2014). The Development of Historical Dramatic Structure in Radw Ashour's Fiction from"1992-2010", Middle East University, Amman, Jordan.

Geibel, Emanuel, Fern im Süden, in: Seibert, C. G. (1860). Ueber ein charakteristisches Element in der Lyrik Emanuel Geibels. Ein Vortrag.

Gelhard, D. (2008). " Mit dem Gesicht nach vorne gewandt": erzählte Tradition in der deutsch-jüdischen Literatur (Vol. 17). Otto Harrassowitz Verlag.

Hanafy, H. (2020). Alternative Histories: Renegotiating Colonial Modernity and the Novel Form in Radwa Ashour's A Part of Europe (Qit'a min Urubba, 2003)". Journal of scientific research in Arts language and Literature, Bd. 21, 1-32.

Hucheon, Linda. A Poetics of Postmodernism: History, Theory, Fiction. New York \& London: Routledge, 2003. 
Lukács, G. (1983). AT” örténelmi Regény: The Historical Novel. U of Nebraska Press.

Louayene, A. (2013). Pathologies of Moorishness: al-Andalus, narrative, and" worldly humanism".

Mohamed, A. K. S. (2015). Chronische Erkrankungen und Überlebensversuche in der deutschen und ägyptischen Gegenwartsliteratur. Eine vergleichende Untersuchung der autobiographischen Romane" Leben" von David Wagner und" Athqal min Radwa" von Radwa 'Aschur. Philology, 63(1). 271- 302.

Nünning, A. (Ed.). (2013). Metzler Lexikon Literatur-und Kulturtheorie: Ansätze-Personen-Grundbegriffe. Springer-Verlag.

Ofner, V. (2004). Die Historischen Romane Robert Neumanns (Doctoral dissertation, uniwien).

Ojeda Arnal, C. (2019). MEMORICIDIO EN GRANADA DE RADWA ASHUR. Miscelanea de Estudios Arabes y Hebraicos, 68. 295 - 322.

\section{Arabischsprachige Literatur}

- - الطبري، محمد بن جرير، 310 هـ (تاريخ الرسل والملوك، تحقيق محمد ابو الفضل، مطابع

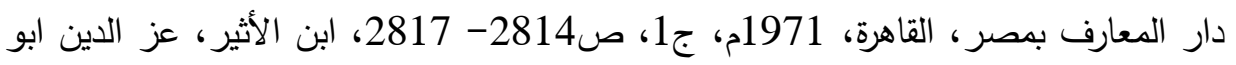

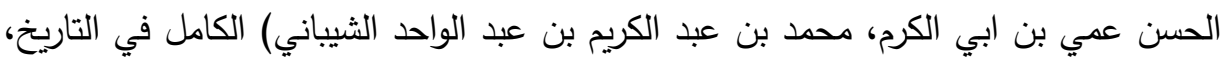

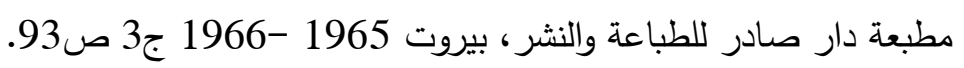

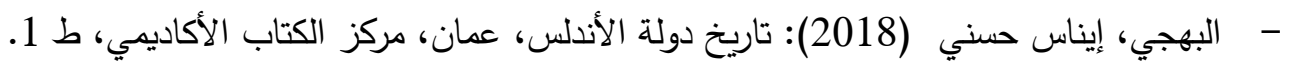

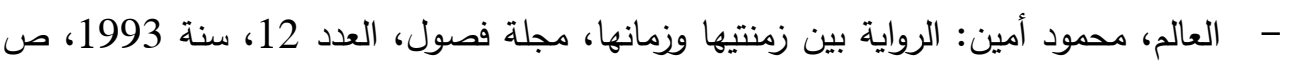

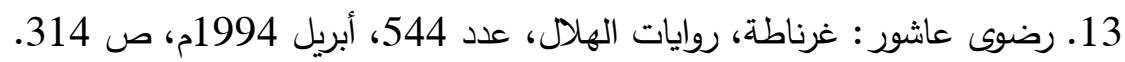

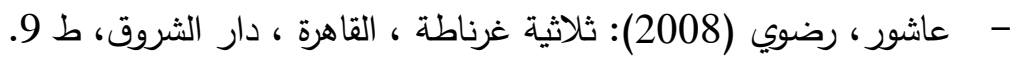

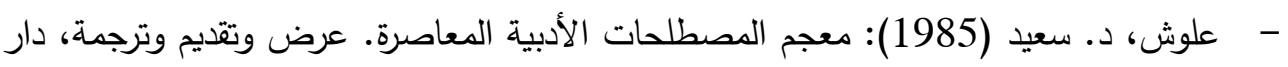

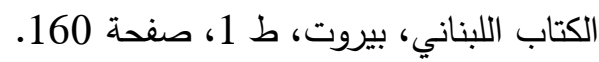


Research in Language Teaching Vol. 2: October $2021 \quad$ Issue № 17

- معجم المصطلحات العربية في اللغة والأدب (ص، 184) في طبيل ، محمد محمد حسن

تحولات الرواية التاريخية في الأدب العربي ، صفحة 3.

- - قط، عبد الحميد عبد العظيم (1982): بناء الرواية في الأدب المصري الحديث، دابه دار المعارف،

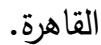

